\title{
ELECTROMAGNETIC FIELD SIGNATURES IN THE OPTICAL SPECTRUM
}

\author{
T. Limperis and D. Goerge \\ Willow Run Laboratories \\ The Institute of Science and Technology \\ The University of Michigan \\ Ann Arbor, Mich.
}

\section{INTRODUCTION}

In the past two decades many new high-performance sensors have evolved that are responsive to portions of the optical spectrum from $0.3 \mu$ (the ultraviolet region) to $1000 \mu$ (beginning of the passive microwave region). These optical sensors have been exploited to some extent in scientific space applications. For example, horizon scanners operating in the infrared region have been used for attitude control of manned spacecraft as well as of some satellites. Tiros meteorological satellites view the earth in several spectral regions simultaneously to provide visible imagery of cloud tops and infrared thermographs that indicate temperature distributions in the earth's atmosphere. The Nimbus satellite contains an infrared grating spectrometer to provide a detailed spectral analysis of the earth's atmosphere. The Mariner flyby vehicle included in its payload an infrared radiometer to scan the surface of Venus in order to determine the water vapor content of its atmosphere by sensing the radiation in and near a water-absorption band.

Each of these applications depends upon spectral, and in some cases spatial, information. This is not the only usable information, however. Electromagnetic radiation arriving at a satellite-borne sensor contains information in five basic forms. These forms include both the spectral and the spatial distributions of radiation intensity (i.e., size and shape), the temporal distribution of radiation intensity, the resultant polarization angle of the electric and magnetic field vectors, and the phase. The basic concepts involved are discussed briefly in the next section.

\section{BASIC CONCEPTS}

The irradiance $\mathrm{H}$ (with units of watts $\mathrm{x} \mathrm{cm}^{-2}$ ) on the aperture of an optical sensor can be written as follows:

$$
\mathrm{H}=\mathrm{H}(\lambda ; \mathrm{t} ; \mathrm{p} ; \theta, \phi ; \Delta \mathrm{x}, \Delta \mathrm{y} ; \mathrm{x}, \mathrm{y})
$$

This representation shows the parametric or functional relationship of the important quantities that affect the incident radiation flux. The wavelength $\lambda$ is perhaps the most important variable. For blackbodies and graybodies, $H$ will vary with $\lambda$ according to Planck's law. There, is no perfect blackbody, and so all materials will deviate to some degree from this ideal distribution; in particular, some materials may have very distinct emission lines or bands. 
The irradiance also will vary with time, on both short- and long-term bases. Some things, such as regions on the moon that have passed from sunlight to shadow, may exhibit significant variability on a millisecond basis; other substances, such as foliage, exhibit diurnal and seasonal variability. Radiation is also polarized to some degree, depending upon the regularity and composition of the materials in the field of view. Also, the radiation depends upon the aspect angles $\theta$ and $\phi$ at which an object is irradiated and viewed, upon the instantaneous groundpatch size $\Delta x, \Delta y$, and upon the observed groundpatch coordinates $x$ and $y$. The change in irradiance in relation to $\lambda ; t$; $\theta ; \phi ; \mathrm{p} ; \Delta \mathrm{x}, \Delta \mathrm{y} ;$ and $\mathrm{x}, \mathrm{y}$ from one class of objects to another is the basis for determining signatures.

The detector's input signal is a function only of the scene's composition, the source (if reflectance is important), and the intervening atmosphere. In terms of the spectral radiance, $N_{\lambda}$, of a particular object it is written mathematically as follows:

$$
\mathbf{H}_{\lambda}=\mathbf{N}_{\lambda} \frac{\mathbf{A}_{\rho} \Omega}{\mathbf{A}_{c}}
$$

where $A_{o}$ is the area* of the particular object, $A_{c}$ is the area of the collector, $\Omega$ is the solid angular field of view and $N_{\lambda}$ is the radiance, or the watts $/ \mathrm{cm}^{2}$. sterad- $\mu$, emanating from the observed objects.

In general, $N_{\lambda}$ contains two terms that dictate its value: the amount of emitted radiation and the amount of reflected radiation. The emitted radiation depends upon the emittance $\epsilon$ from the object and its temperature $T$, and the reflected radiation depends upon the source of natural radiation and the reflectance $\rho$ from the object. Both emittance and reflectance from the object depend upon environmental parameters, the conditions affecting the object at the moment (surface structure, wetness, etc.), and material constituents.

The self-emitted radiation from natural objects is described by Planck's radiation law modified by the spectral distribution of the object's emittance, as shown by the following equation:

$$
N_{\lambda}=K_{1} \epsilon_{\lambda} \lambda^{-5}\left[\exp \left(\frac{K_{2}}{\lambda T}\right)-1\right]^{-1}
$$

Where $K_{1}$ and $K_{2}$ are constants, $\epsilon_{\lambda}$ is the spectral emittance, and $T$ is the object's temperature.

Radiation reflected from an object is ultimately derived from either a natural or an artificial source. Natural sources include illumination from the sun or sky, whereas lasers and radar transmitters are artificial sources. For diffusely reflecting objects, the radiance caused by reflection is given by

$$
\mathrm{N}_{\lambda}=\mathrm{K}_{3} \rho_{\lambda} \mathrm{H}_{\lambda}
$$

where $K_{3}$ is a constant, $\rho_{\lambda}$ is the spectral-directional reflectance, and $H_{\lambda}$ is the spectral irradiance incident on the object from the natural or artificial

*This assumes that the object fills the sensor's field of view. 
source. This equation demonstrates the importance of the spectral shape of the source radiation. Substituting Equations 2 and 3 into Equation 1 gives an expression for the radiant flux at an optical sensor resulting from radiation reflected and emitted by the object. Both terms in the expression obtained by this substitution should contain a multiplicative factor $\tau(\lambda)$ to account for the spectral dependence of transmission through the intervening atmosphere.

The five informational modes that exist in this irradiance value are discussed individually in the subsequent sections, where there are also brief descriptions of some measurements. Also included is a description of the Target Signatures Analysis Center at The University of Michigan, which maintains a current collection of electromagnetic data on commonly occurring materials.

\section{SPECTRAL INFORMATION}

The spectral distribution of irradiance is determined by an object's material constituents, its surface structure, and its temperature. It is also greatly influenced by the characteristics of natural radiation illuminating the object. The spectral dependence of the intervening atmosphere's transmission, of course, acts to further influence the spectral distribution as it is observed with a remote sensor.

An example of spectral information that may be exploited as an object's signature is shown in FIGURE 1, which shows the spectral reflectances of polished minerals determined by G. R. Hunt and J. W. Salisbury at the Air Force Cambridge Research Center. ${ }^{1}$ The vibrational resonance frequencies (restrahlen bands) of silicate rocks and minerals (the principal constituents of the earth's surface) all occur at wavelengths longer than $8.0 \mu$. The relatively high reflectance exhibited by these minerals in the well-known 8.0 -to-14.0- $\mu$ window of the earth's atmosphere may be particularly useful. An infrared

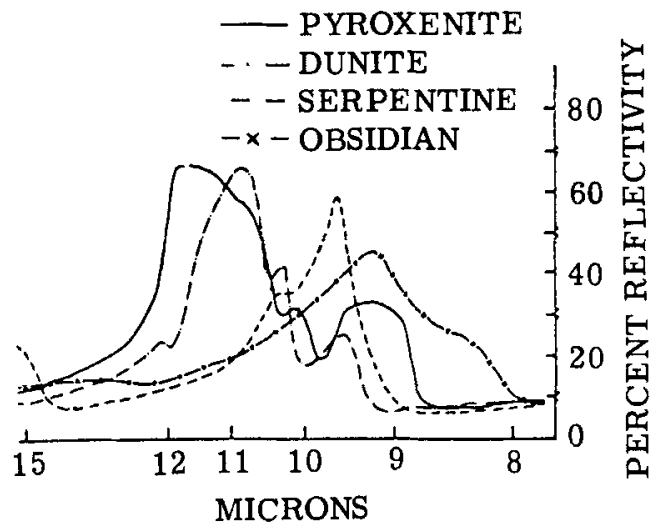

FIGURE 1. Reflection spectra of polished rock samples (Figure copyright 1964 by the American Association for the Advancement of Science). 


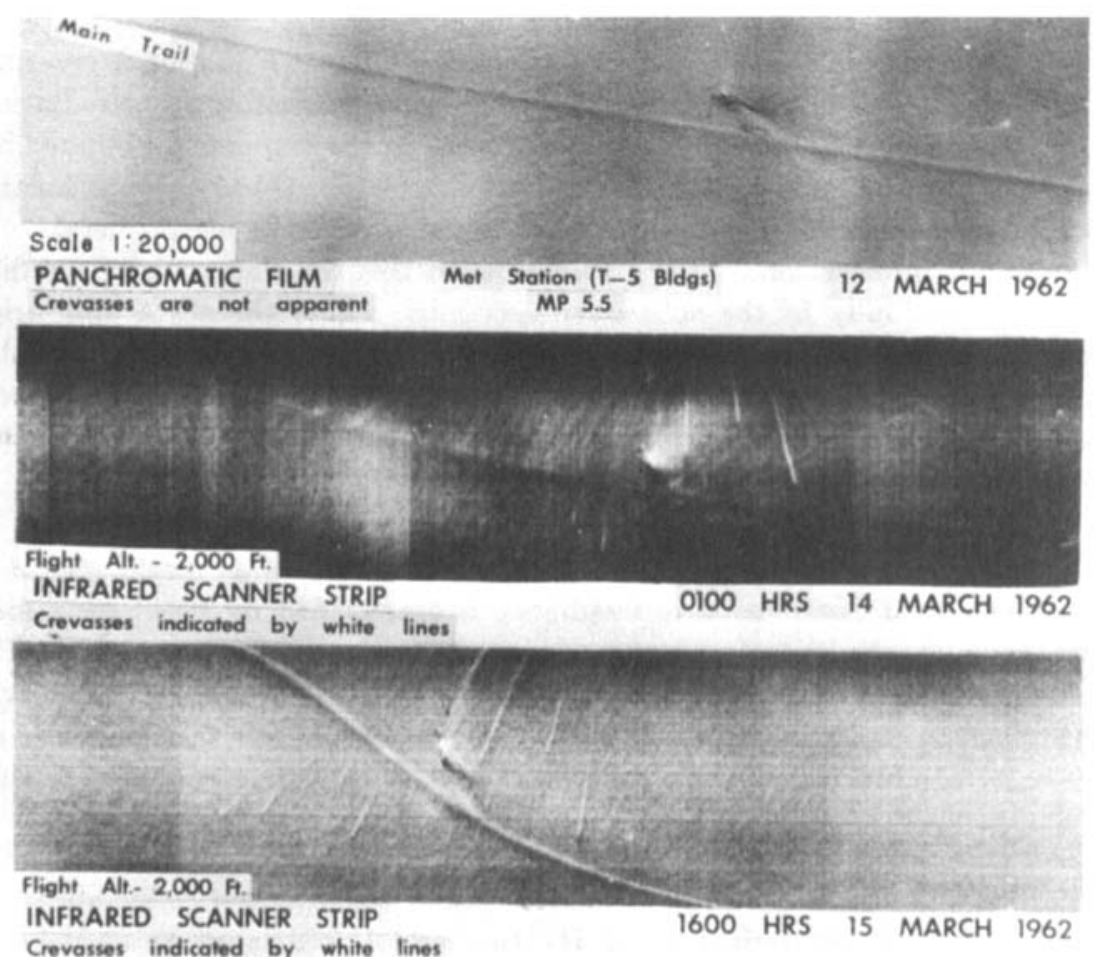

FIGURE 2. Detection of snow-covered crevasses by infrared sensor. ${ }^{7}$ A conventional aerial photograph (top) fails to give any clue to the hidden crevasses revealed by infrared in the two lower photographs. (U. S. Army Cold Regions Research and Engineering Laboratory).

sensor operating in two narrow spectral intervals, one centered at $9.7 \mu$ and the other at $9.0 \mu$, could serve to detect deposits of serpentine. The signal voltages from the two channels could be subtracted to discriminate this mineral from others. Similar measurements of other minerals were made by W. W. Coblentz in $1906 .{ }^{2}$ The possibility of using remotely-sensed spectral information to detect minerals is being investigated by W. Hovis ${ }^{3,4}$ and R. J. P. Lyon ${ }^{5}$ of NASA.

A rather interesting use of spectral information for remote detection of mineral deposits has been demonstrated by A. R. Barringer. ${ }^{6}$ In this case, such inorganic gases and vapors as sulfur dioxide, mercury, and iodine (which emanate from mineral deposits) are detected by operating the sensor in the spectral region containing the strong absorption bands of the emanating gases.

Another example of information gained by judiciously selecting the spectral regions of operation is given in FIGURE 2. This figure shows a conventional aerial photograph and two infrared strip maps obtained in 1962 when the U.S. Army and The University of Michigan conducted a joint study in the 
Arctic on the feasibility of detecting snow-covered crevasses using infrared sensors operating in the 4.5 -to-5.5- $\mu$ band. $^{7}$ The infrared maps reveal a network of crevasses on the Greenland ice cap which cannot be seen in the aerial photograph. The snow-covered crevasses were warmer than the surrounding snow, resulting in a considerable contrast.

When determining the spectral region of sensor operation, one must take into account the transmittance of the atmosphere. FIGURE 3 is a result of computing the earth's atmospheric transmission for two different meteorological conditions, to indicate the variations introduced by changes in the concentration of atmospheric constituents. These computations were made for an aircraft altitude of $10,000 \mathrm{ft}$., which is the reason for the absence of the ozone absorption bands at $9.7 \mu$. At satellite altitudes, the water and $\mathrm{CO}_{2}$ bands would broaden and deepen, and the ozone band would appear.

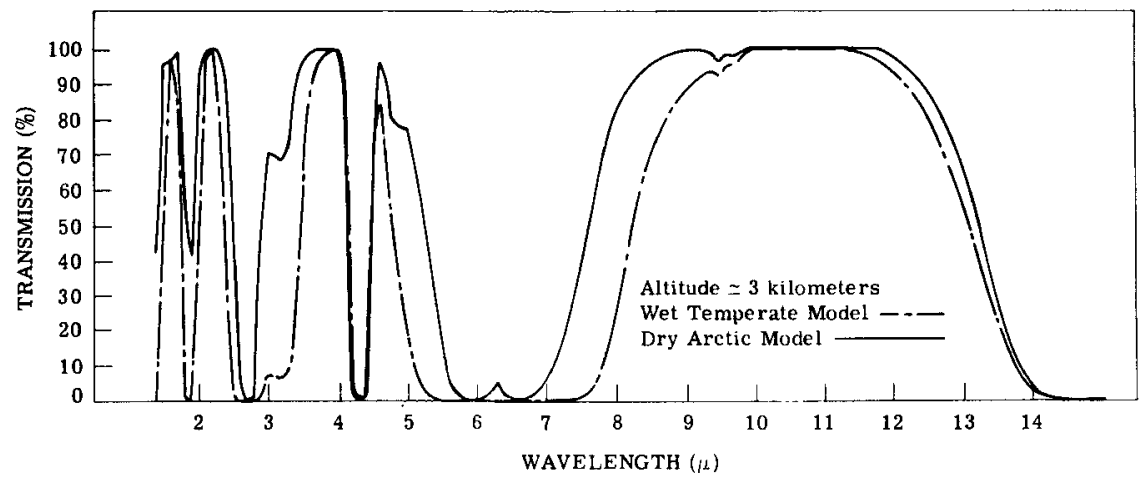

FIGURE 3. Atmospheric transmission as a function of wavelength with wet temperature and dry Arctic models. (The computer program to generate these data was written by D. Anding of the Infrared Information Analysis Center (IRIA) at The University of Michigan.)

\section{SPATIAL INFORMATION}

Spatial information exists in the size and shape of objects, which are obvious characteristics when viewed in the visible spectrum. However, in other portions of the spectrum, size and shape are not determined by the object's geometry alone; the material composition and the structure of its surface, as well as the temperature distribution across the object's surface must also be considered.

Much information about a scene can be obtained by merely taking a picture of it with an ordinary camera or scanning device. Usually operating in rather a broad spectral band, the sensor observes a given patch of ground made up of several constituents. Each constituent emits and reflects in accordance with its individual optical properties, and the radiation-intensity distribution is photographically recorded to preserve the spatial information of the 
scene. FIGURE 4, an example of such imagery, was obtained during an infrared survey program of Hawaiian volcanoes in $1963 .{ }^{8}$ The upper picture is a conventional aerial photograph of the Kilauea summit area. The lower one is an image of the same area taken with an infrared scanner operating in the 4.5-to-5.5- $\mu$ region at an aircraft altitude of 1800 meters. The infrared image, obtained at night, indicates the relative temperature distribution of the Kilauea summit area. Halemaumau, point $A$ in the lower picture, has the highest apparent temperature of the thermal anomalies associated with Kilauea. This site has been the scene of repeated volcanic activity, the most recent eruption at this site having been in 1954. The less centralized thermal anomalies are due to steam exuding from a pattern of cracks on the caldera floor. Point B in the lower picture is a cinder cone formed during a 1959 eruption. Areas 1 and 2 just to the right indicate that there are differences in the apparent surface temperatures of the lava lake, although there are no known corresponding compositional differences. Thus the two pictures provide a relief map of the area in the visible spectrum, showing the shapes of the crater and other features, and a thermal map of the same area, showing the shape and size of the hot zones.

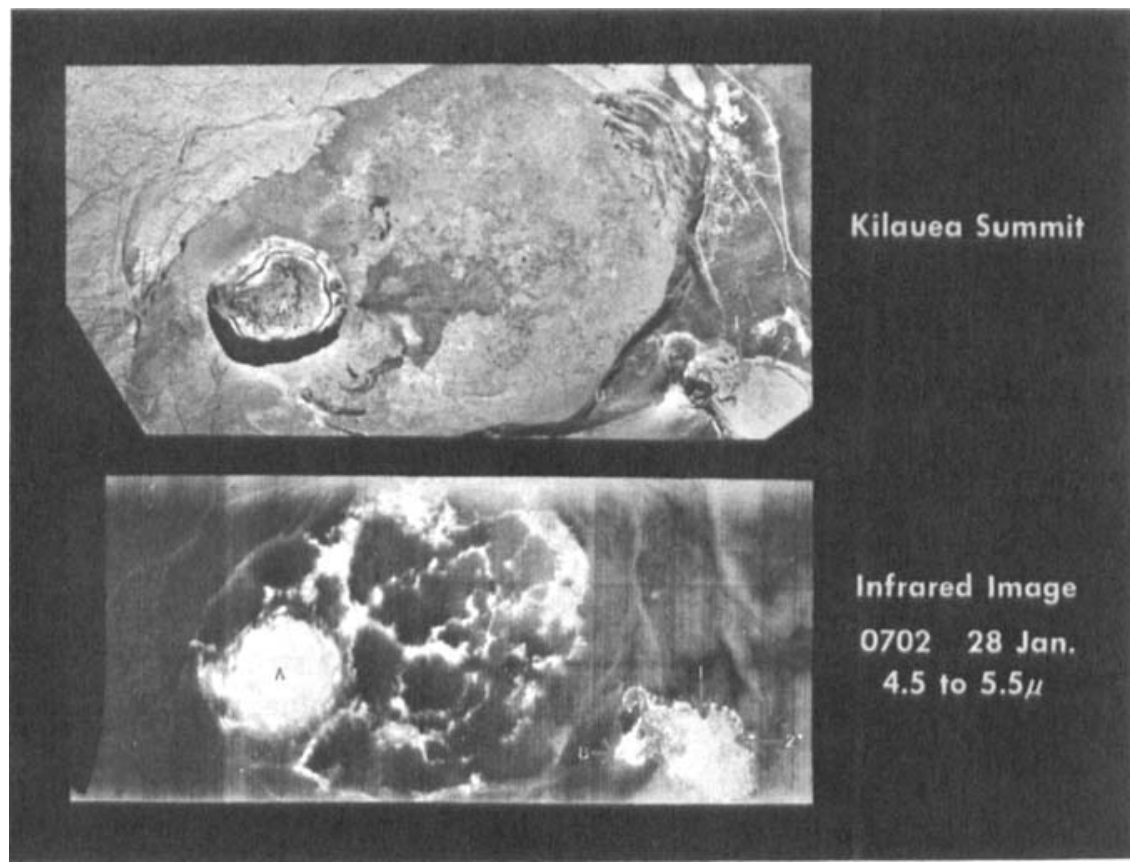

FIGURE 4. Kilauea summit area. (a) Conventional aerial photograph. (b) Infrared image. (Figure copyright 1964 by the American Association for the Advancement of Science). 


\section{TEMPORAL INFORMATION}

Temporal effects are mostly due to diurnal and seasonal variations that affect the material composition of objects; two examples are the material changes in plants caused by the destruction of chlorophyll and other large molecules, and the snow cover in the winter months. Also affected by seasonal and diurnal variations are temperatures of objects and atmospheric constituents.

As an example of temporal information, FIGURE 5 shows the variability in the spectral reflectance of an oak leaf observed at several different times of year, as measured by H. J. Keegan, et al., ${ }^{9}$ at the National Bureau of Standards. The increasing numbers in each set of curves represent subsequent measurements. These data indicate that if careful selection is made of one or
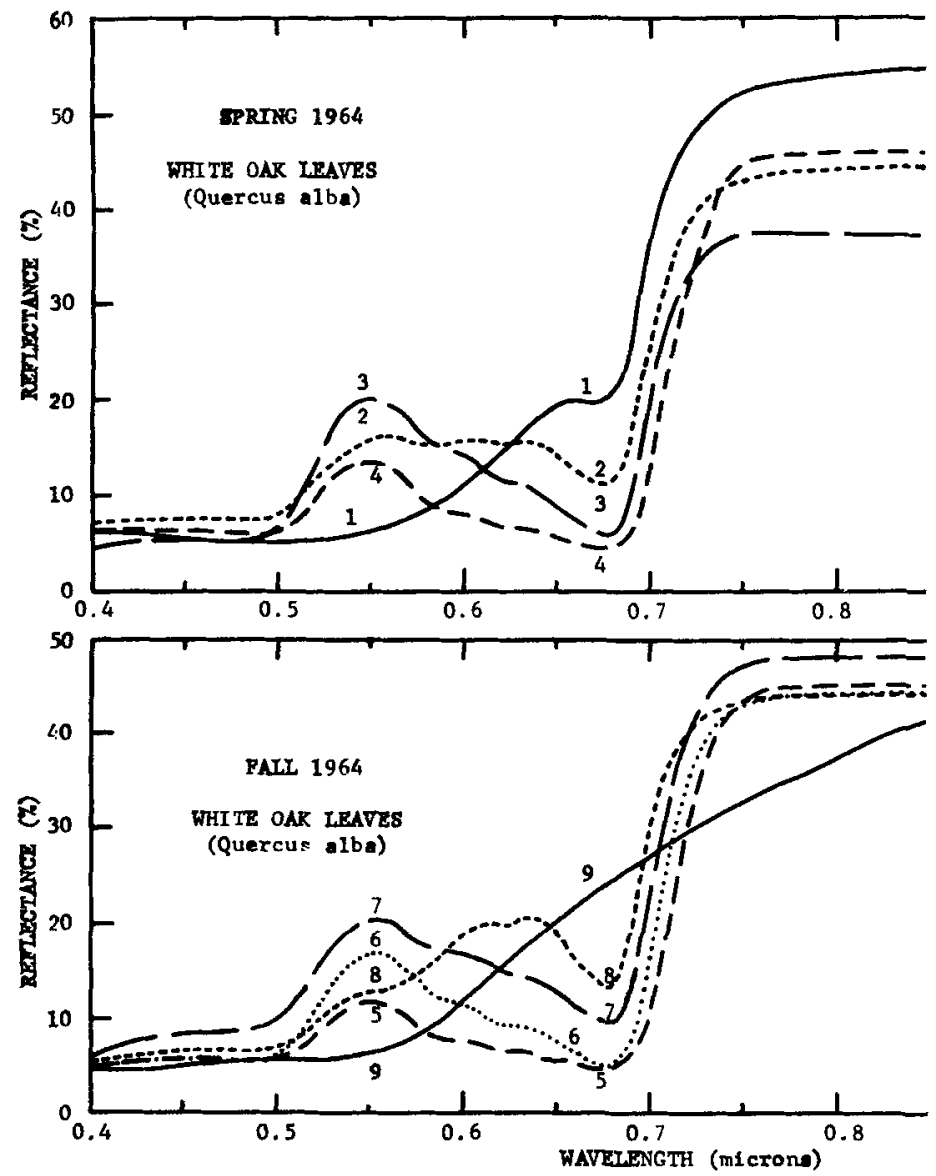

FIGURE 5. Spectral directional reflectance of white oak leaves. (a) Spring 1964. (b) Fall 1964. ${ }^{9}$ 
more spectral intervals of operation, green plants may be detected by observing the area repeatedly during the year. Studies of the temporal effects on the electromagnetic properties of a variety of background materials are currently in progress at The University of Michigan.

\section{POLARIZATION INFORMATION}

Polarization of the radiation emanating from an object offers another important avenue of information. Although polarization in the microwave spectrum has been studied extensively, the optical portion of the electromagnetic spectrum remains relatively unexploited. K. L. Coulson of the University

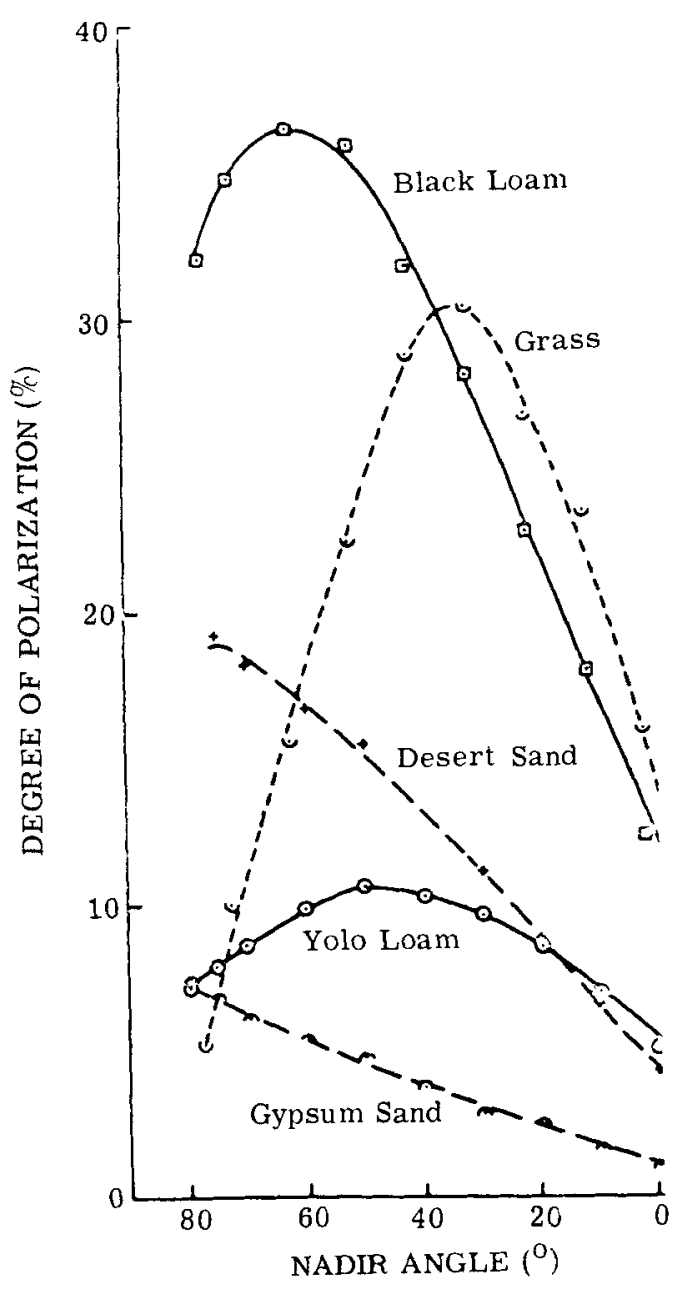

FIGURE 6. Polarization of radiation reflected from various materials $(\lambda=4920 \AA$ and angle of incidence at $\left.53.1^{\circ}\right)^{10}$ 
of California has measured the degree of linear polarization of visible radiation reflected from soils and sands. ${ }^{10}$ FIGURE 6 is a plot of the degree of linear polarization of radiation reflected in the plane of incidence from a group of sands and soils as a function of angle from the surface normal (i.e., nadir angle). The degree of polarization varies significantly from one material to another. There is a definite maximum in the curves for soils and grass, while no pronounced maximum exists for sands. As part of the same investigation, Coulson has made directional reflectance measurements on these quantities and observed that large degrees of linear polarization occur for materials of low reflectance, and vice versa.

A. Dollfus has observed significant polarization in the visible radiation reflected from Mars. ${ }^{11}$ From his information, it has been concluded that the reddish-yellow desert areas are covered with powdered limonite, a hydrated iron oxide. This is the only known mineral that exhibits the same polarization and spectral characteristics as those observed from Mars.

The University of Michigan has conducted cursory airborne measurements to determine whether or not polarization would be a useful reconnaissance tool. In one experiment, two Graflex cameras with Plus-X film and an analyzer, which operated over the $0.3-$ to- $0.7-\mu$ region, were mounted on an aircraft. The polarization axes of the two analyzers were placed in position at $90^{\circ}$ to each other (FIGURE 7); the cameras were operated simultaneously. This experiment was designed to investigate the existence of polarization in reflected radiation from natural and artificial objects as viewed from an aircraft. FIGURES 8 and 9 are examples of the photographs taken. FIGURE 8 indicates both the texture and gross shape-discrimination effects. The water, which is a specular reflector in the visible region, has a considerably different intensity in the two photographs. On the other hand, the image of a small ice sheet on the surface of the lake (which is equally flat, but has a different surface texture) is unpolarized and therefore is not as severely affected by the polarization filters. The white patches at the bottom center of the twin photographs are snow, which is a diffuse reflector. An extremely interesting feature shown in FIGURE 9 is the contrast inversion occurring at the tip of the island (indicated by arrows). It implies that the radiation from the tip is polarized in the plane of incidence. The composition of that portion of the island and its state at the time the photographs were taken are unknown.
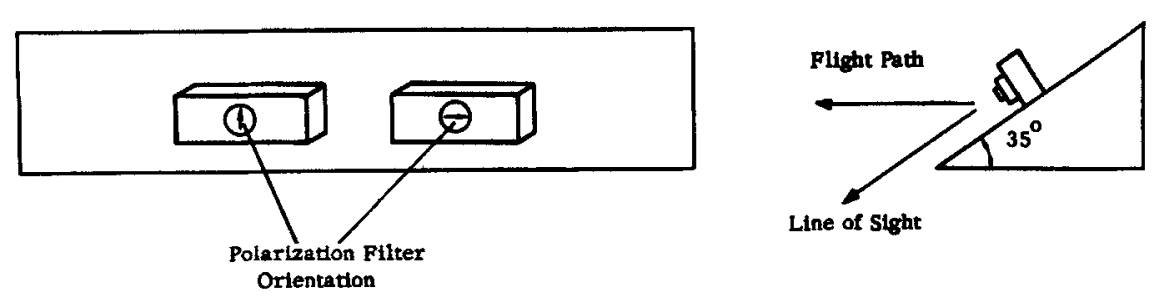

FIGURE 7. Schematic of camera arrangement. 


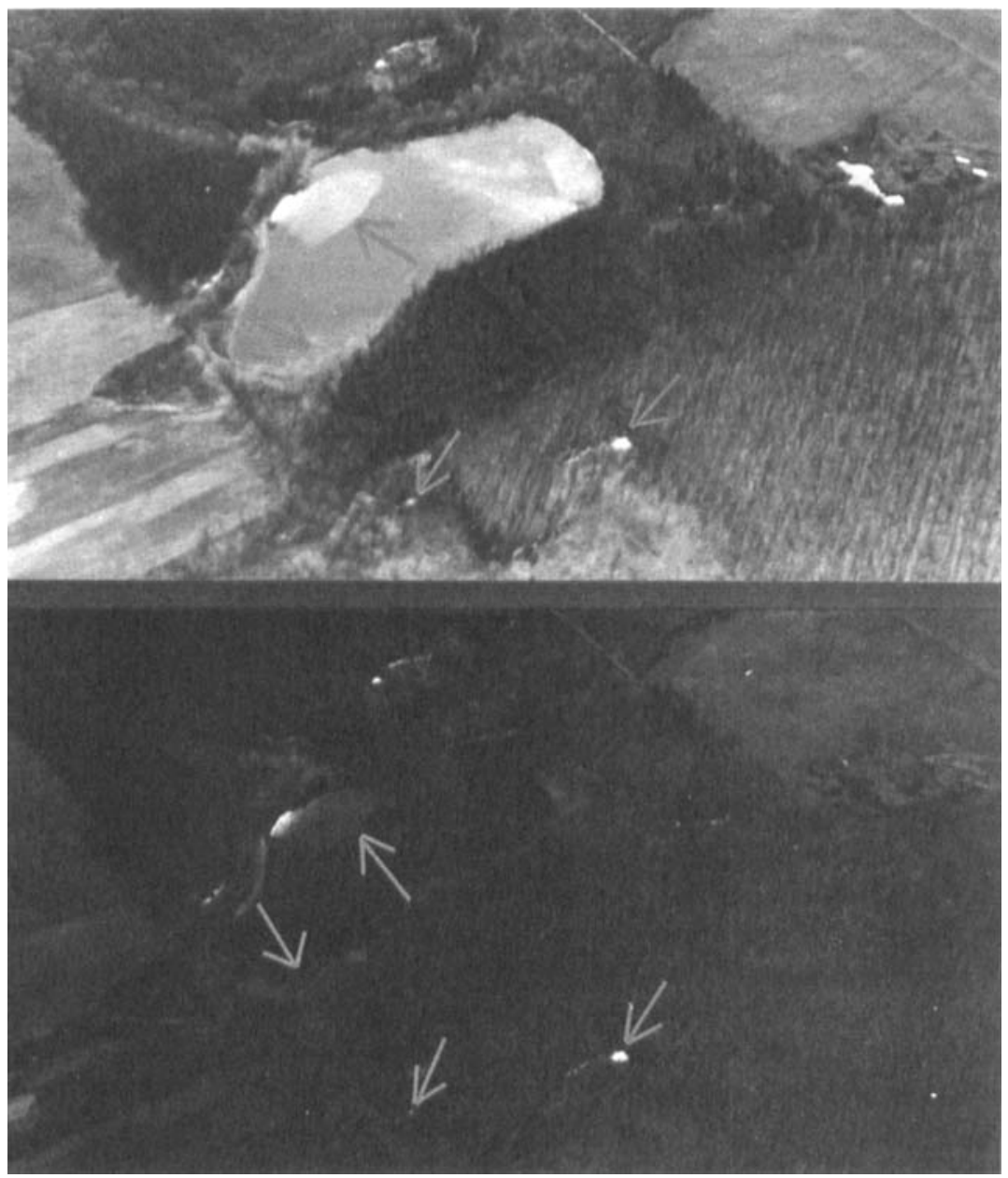

FIGURE 8. Lake and thin-ice sheet photographed with two orientations of a polarization analyzer.

These photographs were taken with the aircraft flying into the sun for the greatest amount of specularly reflected light. When skies are overcast, however, such preferred flight paths are not necessary because there exists an essentially hemispherically emitting source (the illuminated clouds) which distributes the specular reflections to all angles.

These preliminary data indicate that reflected radiation viewed from aircraft platforms does contain polarization components that can be used for discrimination; since the radiation viewed was reflected, there seems to be no obvious reason why this technique cannot be extended to longer wavelengths. 


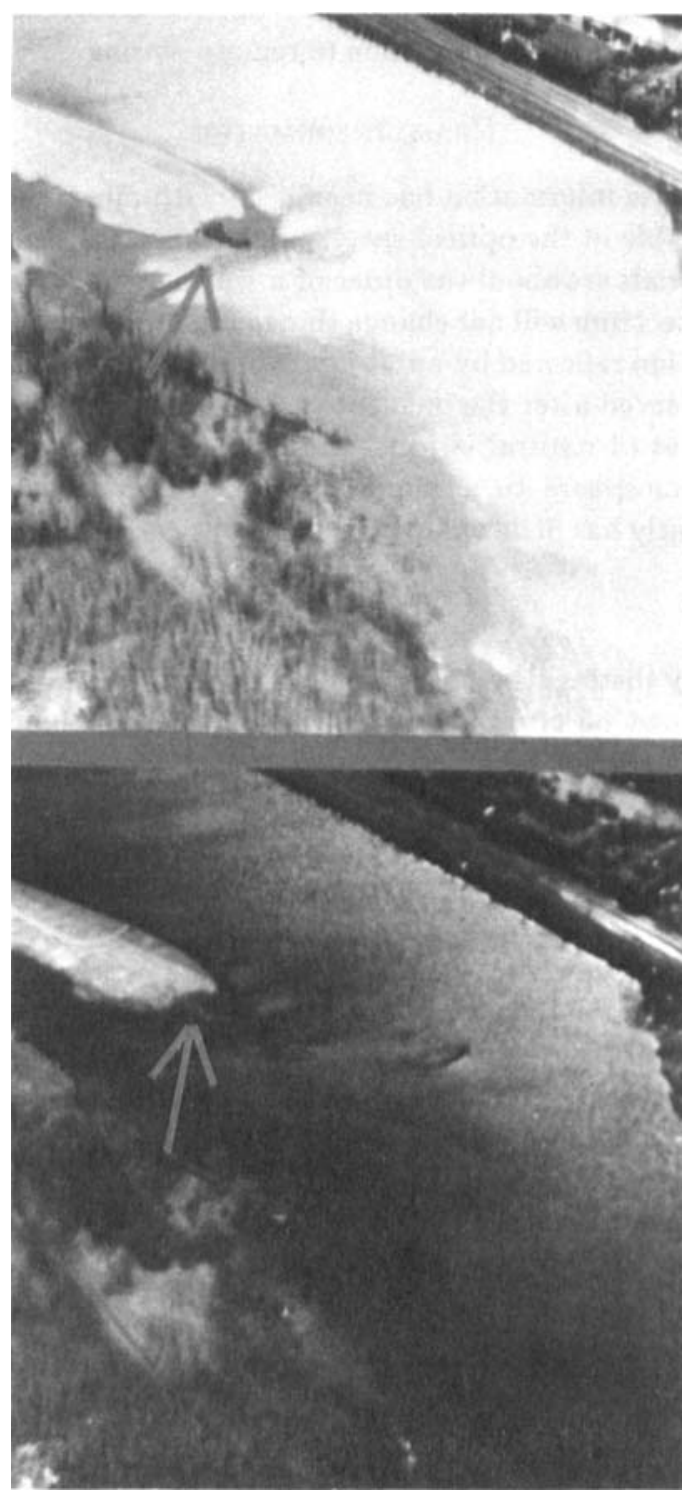

FIGURE 9. River photographed with two orientations of a polarization analyzer.

With regard to polarization that is other than linear, little is known about the degree of elliptical polarization characteristics of materials. Highly refined optical materials have been studied extensively for elliptical polarization in the reflected radiation to determine the real and imaginary parts of the index of refraction. These efforts have led to a relatively new technology called ellip- 
sometry. However, to the authors' knowledge, no effort has been made to study natural materials for application to remote sensing.

\section{Phase Information}

Although phase information has been used with microwave sensors, it has not been applicable in the optical spectrum, because the surface irregularities of natural materials are about the order of a wavelength. The advent of lasers in the optical spectrum will not change things much. The phase of the electromagnetic radiation reflected by an object illuminated with coherent radiation will not be preserved after the incident radiation has been scattered from the irregular surfaces of natural objects and has traversed a sizable pathlength through the atmosphere to a remote sensor. Information derived in this manner apparently has little value in terms of optical discrimination.

\section{ConClusion}

To optimally distinguish an object or class of objects from a background, consideration must be given to all of the information modes of radiation; namely, spectral, spatial, temporal, polarization, and phase (where applicable). This is true both for specialized sensors, which are designed to detect only the presence of one kind of object, and for sensors that gather general information and are used to record much information about the observed scene. In order to design the specialized sensor, information about the object of interest and the background is needed, whereas for the general system, the same information is needed to interpret the data output. Unfortunately, basic information about these characteristics for various objects of interest in space exploration is meager; more measurements are required. Most of the existing data have been collected and placed in a library at the Willow Run Laboratories of The University of Michigan. This library is described in the next section.

\section{Signature ANaLysis}

Signatures are currently being studied at the Willow Run Laboratories of The University of Michigan. This study involves collecting, storing, and analyzing existing data concerning electromagnetic characteristics of objects. At the onset of this study, documents were collected from several sources, such as the Defense Documentation Center (DDC) and the Infrared Information Analysis Center (IRIA), and from research laboratories throughout the country. Over 1,000 documents have been incorporated into a well catalogued and indexed library. From these documents, more than 3,500 data curves have been extracted to form the data library. To keep the library current, the literature is scanned for new publications at regular intervals and requests are made for the library to be placed on the automatic distribution lists of those organizations engaged in research on electromagnetic signatures. 
For each document, the bibliographical and cross-referencing information necessary for retrieval is keypunched onto IBM cards, and then posted to magnetic tape for storage. This information, among other things, includes the document's title, authors, corporate author, monitoring agency, sponsor, contract number, publication date, and a library accessions number. Codes are assigned to each document to adequately describe its content and to enable fast computer retrieval by subject as well as by bibliographical information. Data from these requests are added to the data library. These data are digitized in a common format, punched on IBM cards, and stored on a master data tape. The conditions of the experiment, called parameter information, are also itemized, punched on cards, and posted on cards, and posted to tape. Parameter information includes the following data:

(1) Classification of object measured

(2) Date and time of measurement

(3) Location (latitude and longitude)

(4) Altitude

(5) Range

(6) Zenith and azimuth angles of incidence and observation

(7) Type of illumination (natural or artificial)
(8) Obstructions during measurement (snow, haze, fog, etc.)

(9) Temperature of object

(10) Wind speed and direction

(11) Cloud cover

(12) Visibility

(13) Environmental temperature

(14) Dew point

This information is obtained when it is available from the original document.

The bulk of the optical data consists of spectral directional reflectance of target and background materials, although some emittance and polarization data are included.

Several programs for the IBM 7090 computer have been written for the manipulation and analysis of the data. Some of the programs designed for the optical data are:

(1) A program to punch out the optical data on plotting cards. These cards in turn are fed into a mechanical plotter, and the curve may be reproduced to any desired scale in a matter of minutes

(2) A program that will compute the mean, standard deviation, and the mean plus or minus one standard deviation (see FIGURE 10) for any subset of the data

(3) A general search program to enable retrieval of any subset of data specified by subject or by parametric information, or both

(4) A program to determine the number of data curves as a function of wavelength. (The width of the spectral interval may be chosen according to the resolution desired; FIGURE 11 is an example of this kind of histogram. It presents the number of data curves on soils in the collection as a function of wavelength) 


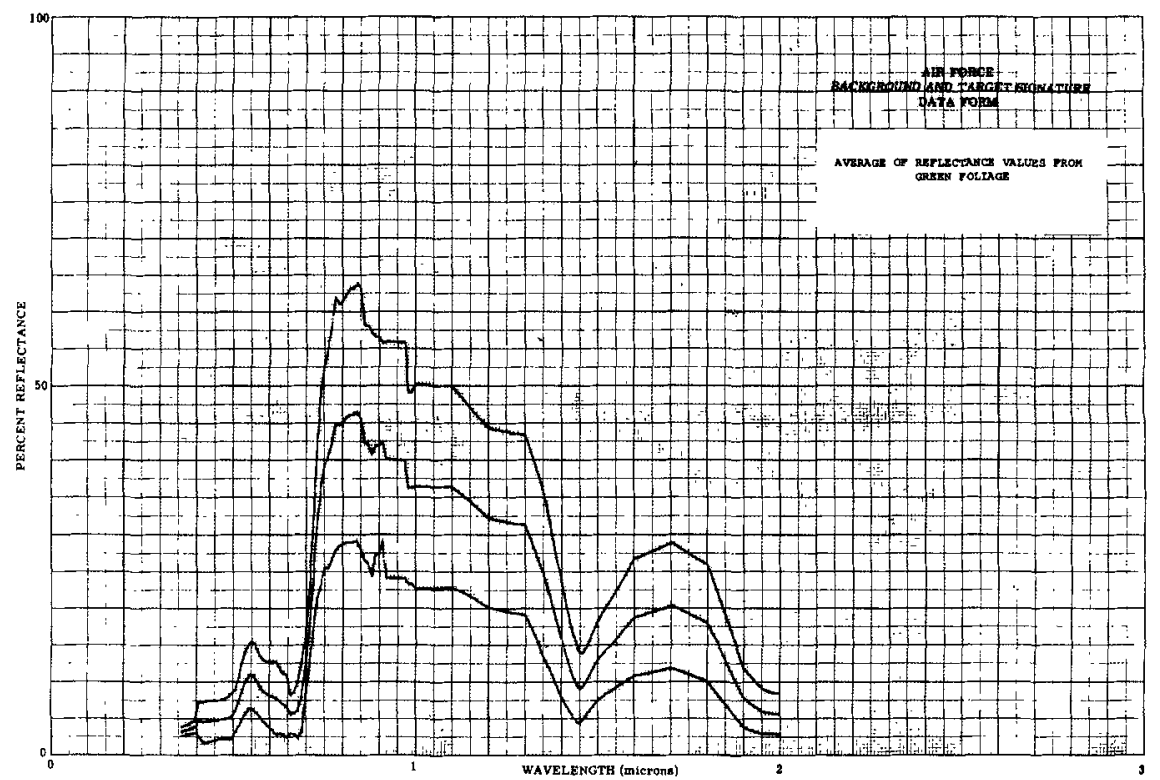

FIGURE 10. The average value and the average value plus and minus one standard deviation of reflectance from green foliage as functions of wavelength.

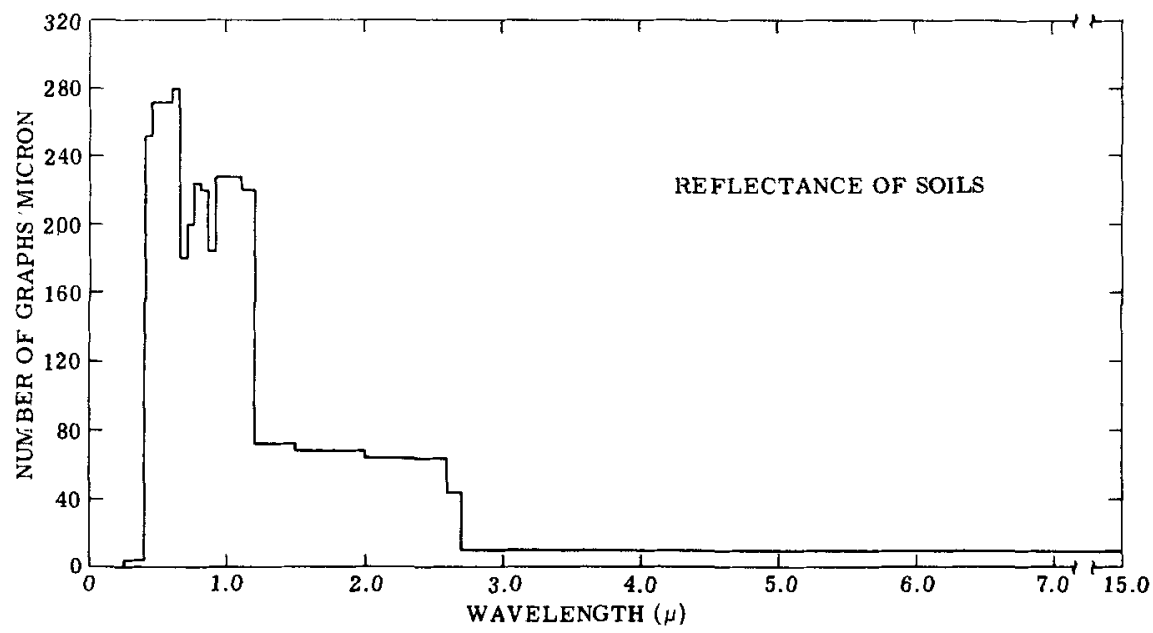

FIGURE 11. Frequency distribution of reflectance data of soils as a function of wavelength.

The primary objective of such a study in signature analysis is to determine optimal techniques for detection, given the object or object-class of interest and the anticipated background. Computations of this kind, using statistical analysis, are now in progress. 


\section{REFERENCES}

1. HUNT, GRAHAM R. \& JOHN W. SALISBURY. 1964. Lunar surface features: midinfrared spectral observations. AFCRL-64-1019, December 1964, AD 456-149. Air Force Cambridge Research Laboratories. Bedford, Mass.

2. COBLENTZ, W. W. 1906. Selective radiation from various solids II. Bull Bur. of Standards 6(3): 301-19.

3. HOVIS, W. A., JR. Infrared reflectivity of some common materials. Applied Optics. (In publication).

4. HOVIS, W. A., JR. Infrared reflectivity of iron oxide minerals. Icarus. (In publication).

5. LYON, R. J. P. 1963. Evaluation of infrared spectrophotometry for compositional analysis of lunar and planetary soils. NASA Technical Note D-1871. April 1963. Washington, D. C.

6. BARRINGER, A. R. 1965. Developments towards the remote sensing of vapours as an airborne and space exploration tool. pp. 279-92. Proc. Remote Sensing Symposium, 1965. Barringer Research, Limited, Rexdale, Ontario, Canada.

7. McLerRAN, JAMES H. 1964. Airborne crevasse detection, Proceedings of the Third Symposium on Remote Sensing of Environment, October 14, 15, 16, 1964. The University of Michigan, Infrared Physics Laboratory, Ann Arbor, Mich.

8. FISCHER, W. A. et al. 1964. Infrared surveys of hawaiian volcanoes. Science 146(3645): 733-42.

9. National Bureau of Standards. 1964. Infrared Optical Measurements. (Semi-annual Technical Report, 1 July through 31 December 1964), Report No. 8626, National Bureau of Standards, Metrology Division, Washington, D. C.

10. COULSON, K. L. 1965. Effect of surface properties on planetary albedo. Thermophysics Specialist Conference, September, 1965. Monterey, Calif.

11. DOLLFUS, AUDOUIN. 1964. Study of the planets by means of the polarization of their light. Thesis, University of Paris, 1955. NASA Technical Translation, NASA TT F-188. 